\title{
ESTRUCTURACIÓN DE LA CADENA DE VALOR DEL CACAO EN EL DEPARTAMENTO DE SUCRE COMO ESTRATEGIA PARA EL CRECIMIENTO ECONÓMICO REGIONAL
}

\section{STRUCTURING THE COCOA VALUE CHAIN IN THE DEPARTMENT OF SUCRE AS A STRATEGY FOR REGIONAL ECONOMIC GROWTH}

\author{
Autor 1. Julieth Alexandra Barón Bayona \\ Economista. Especialista en Gerencia Financiera \\ Docente Universidad Nacional Abierta y a Distancia- UNAD \\ Correo electrónico: Julieth.baron@unad.edu.co
}

Autor 2. Amalio Segundo Otero Tapia

Administrador de Empresas. Magister en Administración de Organizaciones Docente Universidad Nacional Abierta y a Distancia- UNAD ORCID: https://orcid.org/0000-0002-0142-0344

Correo electrónico: Amalio.otero@unad.edu.co

Autor 3. Mario Fernando Zubiria Lara

Administrador público. Especialista en pedagogía para el desarrollo del aprendizaje autónomo - (MBA).

Docente Universidad Nacional Abierta y a Distancia- UNAD

ORCID: https://orcid.org/0000-0002-1392-1728?lang=es

Correo electrónico: mario.zubiria@unad.edu.co

Autor 4. Luis Alejandro Forero Barón

Contador Público. Magister. Magister en diseño y Gestión de Proyectos

Líder de Especialización en Gestión de proyectos

Grupo: Giepe

ORCID: http://orcid.org/0000-0002-8556-5419

Correo electrónico: luisa.forero@unad.edu.co

\section{RESUMEN}

La producción de cacao en el mundo está dividida entre los que se quedan en el primer y último eslabón de la cadena. Para el primer caso se ubican los países en vías de desarrollo como Colombia, Ecuador, Costa de Marfil, Indonesia, entre otros, caracterizados por una baja competitividad y transformación del producto. En el segundo caso se encuentran los 


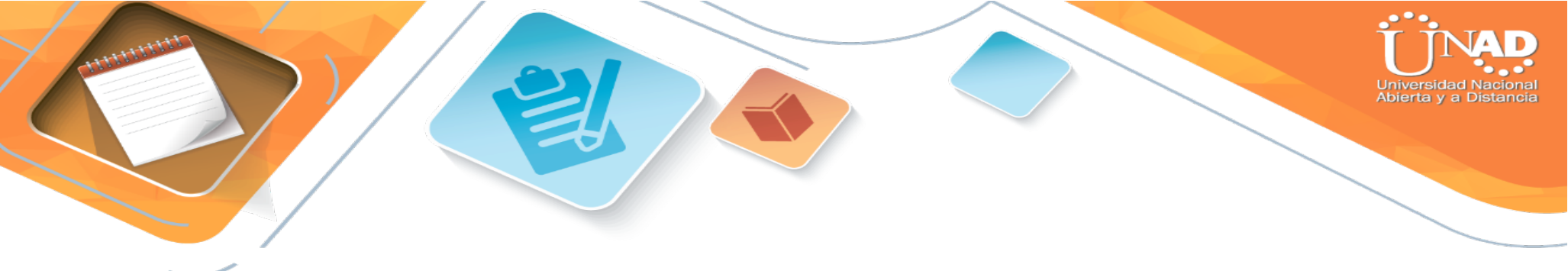

\section{INTRODUCCIÓN}

Las diferentes economías dentro de los países tienden a experimentar cambios considerables en sus estructuras de producción e institucionales como resultado del proceso de globalización la reestructuración productiva y los entornos cambiantes de la economía internacional.

Mayores procesos de flexibilidad del capital y del trabajo, el desarrollo de las tecnologías enmarcadas en la revolución 4.0, han llevado a que las economías mundiales se articulen en redes económicas en todo nivel. Las economías pequeñas y abiertas están dependiendo de las decisiones que se toman en el mercado mundial afectando directamente los mercados locales y nacionales.

El departamento de Sucre no ha sido la excepción a este cambio. Éste cuenta con una riqueza natural, tierras fértiles donde se pueden desarrollar distintas actividades económicas, entre las que se destacan, la agricultura, pesca, ganadería que juegan un papel importante en la economía de la región.

Pese a contar con los anotados recursos, el departamento de Sucre presenta problemas productivos, desempleo y ademas de los problemas sociales (inseguridad, informalidad, etc.), por lo cual es indispensable contribuir al desarrollo de nuevos modelos de negocio que ayuden a mitigar estos flagelos y proponer alternativas de solución a toda esta problemática.

Es aquí donde se propone la articulación de la cadena de valor del cacao como una estrategia para fortalecer a la región que permita construir una industria sólida y completa en torno a este producto, a los campesinos y pequeños productores y que estos logren insertarse a los mercados y puedan conformar su industria dentro de la región.

A través de esta investigación se realiza un análisis del proceso de la cadena de valor del cacao a nivel mundial pasando por la situación a nivel nacional para luego estructurar la cadena de valor en el departamento de sucre. 
derivados del cacao totalizaron en el año 2017 un total de 16.002 millones de dólares creciendo en el periodo del 2012 al 2017 un 3.5\% anual.

Colombia es el décimo país productor de cacao a nivel mundial y solo es superado en Latinoamérica por Ecuador que se encuentra en la $4^{a}$ posición, Brasil en el puesto 7, Perú ocupa el puesto 8, República Dominicana en el 9 puesto. Pero la diferencia la marca la calidad del cacao colombiano que es muy apetecido en el mundo y ha ganado reconocimientos a nivel internacional como uno de los cacaos mas fino y con mejor aroma, distinción que solo se le da al 5\% del grano que se exporta en todo el mundo.

Colombia ademas de tener uno de los mejores granos a nivel mundial en cuanto a calidad, producción de derivados que le dan un valor agregado al grano y con regiones que se han ido especializando en la producción, transformación y comercialización del cacao. Según datos de PROCOLOMBIA aproximadamente hay 14.4 millones de hectáreas aptas para la producción de cacao. Sumado a lo apetecido que es el grano colombiano en el exterior y los programas existentes, que incentivan la siembra en el territorio, hacen posible que el departamento de Sucre pueda insertarse en la cadena nacional.

A nivel nacional se encontraron casos de estudios interesantes para poner en práctica en la región como el caso de Boyacá donde han logrado estructurar una cadena de valor que ha conseguido logros y avances importantes que pueden ser aplicados en el departamento de Sucre logrando, crecimiento económico y competitividad.

Según datos de "la Federación Nacional de Cacaoteros (FEDECACAO, 2017) la producción creció 6,6 por ciento y las exportaciones aumentaron 11 por ciento." (SEMANA, 2018), menciona que el departamento de Sucre hace su aporte a nivel nacional como en el caso de Santander, que aportaba en el año 2017 el 38.1\% del cacao producido en el país. La producción del departamento es pequeña llevado a esta comparación y en donde en relación con la región del Santander, es mucho mas nueva la siembra del grano, pero no deja ser importante, si se tiene en cuenta que en tan solo en el año 2008 el departamento contaba con tan solo 20 hectáreas productivas y en el año 2018 cuenta con 343 hectáreas productivas, con una producción de 209.1 toneladas, teniendo en cuenta que aún hay cultivos que no están en etapa productiva y que, con estos, el área sembrada podría llegar a superar las 500 hectáreas. Otro aspecto importante encontrado es que el rendimiento en el departamento de Sucre es superior al de otras regiones del país como Santander quien 


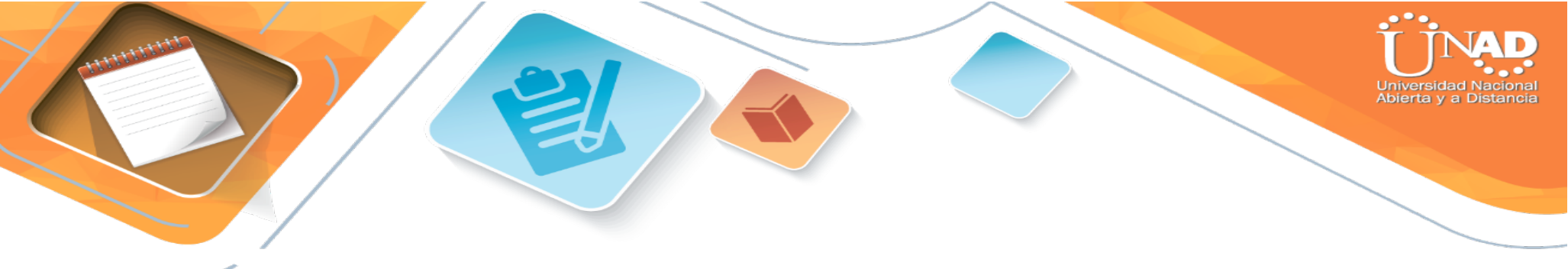

encabeza la lista a nivel nacional en producción, pero quien tiene un rendimiento por hectárea de un $0.52 \%$ mientras que la producción del departamento de Sucre alcanza un $0.7 \%$ lo que es un factor importante para tener en cuenta.

A continuación, se presenta la estructura de la cadena de valor del cacao que potencializará la actividad económica del departamento de Sucre:

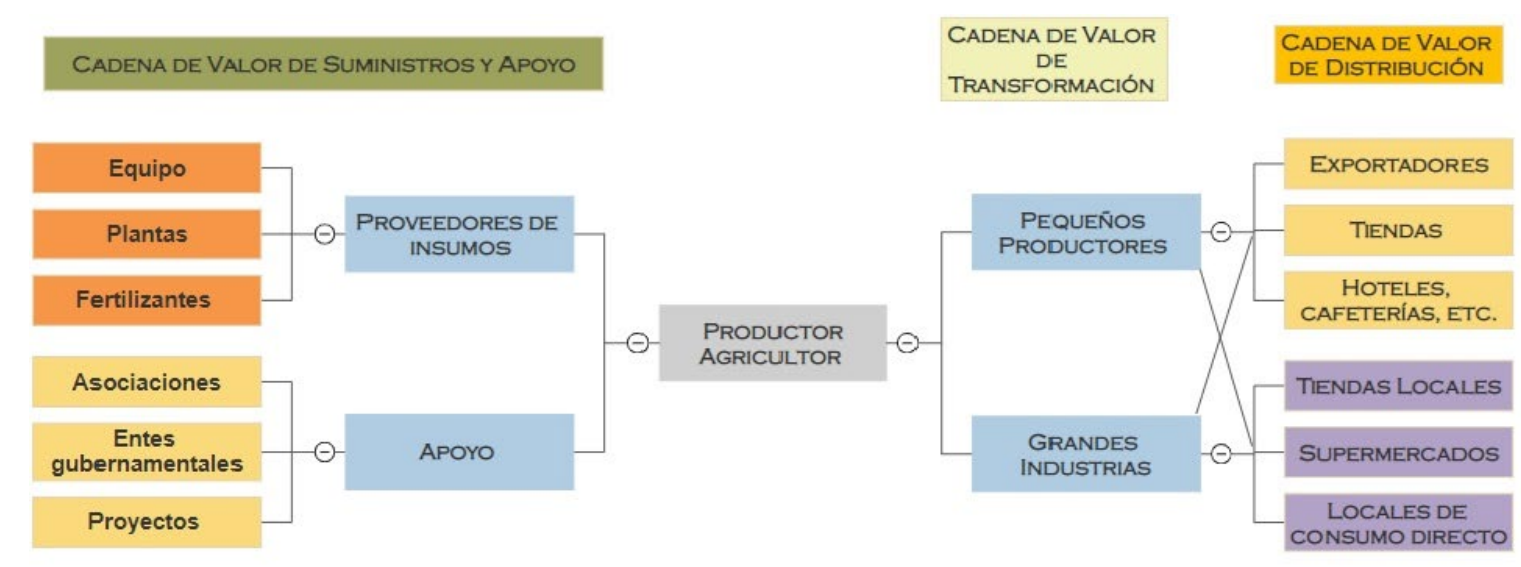

Fuente: elaboración propia.

En la figura se observa el productor o agricultor depende de los suministros y apoyo para llevar a cabo su proceso de cultivo, mantenimiento y cosecha del grano, continúa con la fase de transformación. Este proceso es el que genera la ventaja competitiva, el valor agregado. En esta fase intervienen los pequeños productores y las grandes industrias para llegar a la última fase de distribución con mayor relevancia en la difusión del producto elaborado, inicialmente con el mercado local, luego con las grandes superficies, hoteles y finalmente llegar a exportar el producto.

\section{CONCLUSIONES}

El sector del cacao en Colombia por sus características de calidad y aroma, cuenta con el escenario adecuado para desarrollar actividades que impulsen la competitividad del sector, generar valor agregado en su producto para exportar, este proceso permitirá a los agricultores generar mejor precio, rentabilidad y consolidarse en el mercado a nivel internacional. 
